\title{
Neuromuscular fatigue during a long-duration cycling exercise
}

\author{
ROMUALD LEPERS, NICOLA A. MAFFIULETTI, LUDOVIC ROCHETTE, \\ JULIEN BRUGNIAUX, AND GUILLAUME Y. MILLET \\ Groupe Analyse du Mouvement, Faculté des Sciences du Sport, \\ Université de Bourgogne, 21078 Dijon Cedex, France
}

Received 27 August 2001; accepted in final form 13 December 2001

\begin{abstract}
Lepers, Romuald, Nicola A. Maffiuletti, Ludovic Rochette, Julien Brugniaux, and Guillaume Y. Millet. Neuromuscular fatigue during a long-duration cycling exercise. J Appl Physiol 92: 1487-1493, 2002. First published December 14, 2001; 10.1152/japplphysiol.00880.2001.-The effects of prolonged cycling on neuromuscular parameters were studied in nine endurance-trained subjects during a 5-h exercise sustained at $55 \%$ of the maximal aerobic power. Torque during maximal voluntary contraction (MVC) of the quadriceps muscle decreased progressively throughout the exercise $(P<0.01)$ and was $18 \%$ less at the end of exercise compared with the preexercise value. Peak twitch torque, contraction time, and total area of mechanical response decreased significantly $(P<0.05)$ after the first hour of exercise. In contrast, changes in M-wave characteristics were significant only after the fourth hour of the exercise. Significant reductions $(P<0.05)$ in electromyographic activity normalized to the $\mathrm{M}$ wave occurred after the first hour for the vastus lateralis muscle but only at the end of the exercise for the vastus medialis muscle. Muscle activation level, assessed by the twitch interpolation technique, decreased by $8 \%(P<$ 0.05 ) at the end of the exercise. The results suggest that the time course is such that the contractile properties are significantly altered after the first hour, whereas excitability and central drive are more impaired toward the latter stages of the 5-h cycling exercise.
\end{abstract}

maximal voluntary contraction; electromyography; muscular twitch; activation level; $\mathrm{M}$ wave

NEUROMUSCULAR FATIGUE CAN be defined as any exerciseinduced reduction in maximal voluntary force. During prolonged exercise performed at a constant intensity, there are signs that changes occur in the neuromuscular system as exercise progresses that might influence the efficacy or the pattern with which the contractile machinery is activated. Previous studies of fatigue during long-duration cycling exercise $(>2 \mathrm{~h})$ have focused on the effect of different substrates' utilization on performance (e.g., Refs. 6, 9), but little is known concerning the occurrence of neuromuscular fatigue during such endurance exercise. For example, a reduction of maximal voluntary contractions (MVCs) of the quadriceps muscles has been found after 2-h cycling

Address for reprint requests and other correspondence: R. Lepers, Groupe Analyse du Mouvement, Faculté des Sciences du Sport, Université de Bourgogne, BP 27877, 21078 Dijon Cedex, France (E-mail: romuald.lepers@u-bourgogne.fr). performed at a constant power output (18). This reduction resulted from changes in central and peripheral mechanisms such as decrease of neural input and alterations of the $\mathrm{M}$ wave and isometric muscular twitch.

During prolonged exercise, metabolic changes in the recruited fibers can decrease the force-generating capacity of the skeletal muscle by altering any part of the excitation-contraction coupling process. Because prolonged cycling exercise, performed at a relatively high intensity, will cause progressive muscle glycogen depletion, which alters skeletal muscle metabolism (6, 26 ), it could be expected that peripheral fatigue will occur progressively during such an exercise. Nevertheless, the time course of impairment of quadriceps muscle function and the relative contribution of contractile and neural factors to fatigue during prolonged exercise such as cycling have not been studied. However, muscle glycogen depletion has not been identified as the exclusive cause of fatigue during prolonged exercise (13), and decrements in skeletal muscle power output are not tightly correlated with metabolic changes (1). Consequently, we hypothesize that others factors that are related to the neural drive command lead to muscle fatigue during prolonged exercise. Indeed, reduction in efferent motor command to the active muscles (central fatigue) can also induce a decline in the force development.

The aim of the study was therefore to examine the time course of impairment in central and peripheral processes during a long-duration cycling exercise performed at a relatively high intensity. To estimate the changes that may occur during this prolonged exercise, the neuromuscular (neural and contractile) properties of the quadriceps muscle were evaluated every hour during short interruptions of a 5-h cycling exercise performed at $55 \%$ of the maximal aerobic power (MAP).

\section{METHODS}

\section{Subjects}

Nine endurance-trained male cyclists or triathletes, accustomed to riding for prolonged periods, volunteered to partic-

The costs of publication of this article were defrayed in part by the payment of page charges. The article must therefore be hereby marked "advertisement" in accordance with 18 U.S.C. Section 1734 solely to indicate this fact. 
ipate in this study after they were informed in detail about the nature of the experiment and possible risks. The subjects had regularly trained in cycling for at least $5 \mathrm{yr}$ before the study, and their average weekly training distance during the 2 mo before testing was $200 \pm 50 \mathrm{~km}$. Written informed consent was given by each subject, and a local ethics committee for the protection of individuals gave approval concerning the project before its initiation. The average age of the subjects was $28 \pm 3(\mathrm{SD})$ yr. Average body mass was $72 \pm 6 \mathrm{~kg}$, and average height was $180 \pm 6 \mathrm{~cm}$.

\section{Data Collection and Analysis}

Strength measurement. Instantaneous isometric torque at the knee joint was recorded using a Biodex isokinetic dynamometer (Shirley, NY). Subjects were placed in a seated position and were securely strapped into the test chair. Extraneous movement of the upper body was limited by two crossover shoulder harnesses and a belt across the abdomen. The trunk-thigh angle was $90^{\circ}$. The axis of the dynamometer was aligned with the knee flexion-extension axis, and the lever arm was attached to the shank by using a strap. The knee angle was fixed at $60^{\circ}$ of flexion $\left(0^{\circ}=\right.$ knee fully extended).

Muscular twitch and voluntary activation. To determine the effect of 5-h cycling exercise performed at the same power output on contractile (muscular isometric twitch) and neural (M wave, voluntary activation) properties of the quadriceps muscle, the femoral nerve was electrically stimulated at rest in the seated position as previously described. Isometric tension developed by the muscular twitch was recorded by the isokinetic dynamometer. Electrical stimulations were given by using a high-voltage (maximal voltage $400 \mathrm{~V}$ ) current-constant stimulator (model DS7, Digitimer, Hertfordshire, UK). The femoral nerve was stimulated by using a monopolar cathode ball electrode $(0.5-\mathrm{cm}$ diameter) pressed into the femoral triangle by the experimenter. The site of stimulation was marked on the skin so that it could be exactly repeated between stimulations during the experiment. The anode was a rectangular electrode (Medicompex, Ecublens, Switzerland), $50 \mathrm{~cm}^{2}(10 \mathrm{~cm} \times 5 \mathrm{~cm})$, located in the gluteal fold opposite the cathode. The intensity of the stimulation, which was a $2-\mathrm{ms}$ rectangular pulse, was progressively increased until there was no further increase in the amplitude of the twitch (maximal intensity ranged from 40 to $100 \mathrm{~mA}$ ). We verified that at this intensity the amplitude of the $\mathrm{M}$ waves was also maximal. Once the plateau of the twitch torque was achieved, three stimuli each separated by $5 \mathrm{~s}$ were given. The same stimulus intensity was used during the entire experiment. Maximal twitch torque $\left(\mathrm{P}_{\mathrm{t}}\right)$, twitch time to peak or contraction time, half-relaxation time, and total twitch area were determined from the averaged mechanical response of the three stimuli. Contraction time was measured from the torque onset to $\mathrm{P}_{\mathrm{t}}$ and half-relaxation time was measured from $\mathrm{P}_{t}$ to half the peak value. The torque-time curve was filtered at $100 \mathrm{~Hz}$ with a Butterworth low-pass filter. Total twitch area was calculated by a numeral integration process starting at the beginning of the contraction time until the end of the relaxation period. Maximal rate of torque development and relaxation were calculated as the maximum change of force over time of the filtered torque trace with a length of interval set to $1 \mathrm{~ms}$. Voluntary activation level was estimated by expressing every increment in torque electrically evoked during the maximal isometric contractions (twitch size when superimposed) as a fraction of the amplitude of the response evoked by the same stimulus in the relaxed muscle (twitch size evoked at rest). The activation level (AL) was then estimated according to the formula (15) $\mathrm{AL}(\%)=[1-($ twitch size when superimposed $/$

twitch size evoked at rest)] $\times 100$

EMG recording. Muscle electrical activity [electromyograph (EMG)] of the vastus lateralis (VL) and the vastus medialis (VM) muscles was recorded by means of two pairs of silver chloride circular (diameter $20 \mathrm{~mm}$ ) surface electrodes (Controle Graphique Medical, Brie-Comte-Robert, France) fixed to the right leg. The skin was carefully prepared: low resistance between the two electrodes was obtained $(<5 \mathrm{k} \Omega)$ by light abrasion of the skin, and oil and dirt were removed from the skin with an alcohol swab. Electrodes were coated with electrode gel and fixed lengthwise over the middle of the "belly" of the VM and VL muscles, with an interelectrode distance of $20 \mathrm{~mm}$, and with the reference electrode being fixed on the right wrist. Myoelectric signals were amplified with a bandwidth frequency ranging from 1.5 to $500 \mathrm{~Hz}$ (common mode rejection ratio $=90 \mathrm{~dB}$; impedance input $=$ $100 \mathrm{M} \Omega$; gain $=1,000$ ). Torque and EMG signals were digitized on-line (sampling frequency $2,000 \mathrm{~Hz}$ ) by using a digital computer (IPC 486).

The following parameters were calculated from the $M$ wave of VL and VM muscles: duration, defined as the time interval between the positive and the negative peak of the $M$ wave; peak to peak amplitude; and root mean square (RMS) of $\mathrm{M}$ wave $\left(\mathrm{RMS}_{\mathrm{M}}\right)$ calculated during the time interval corresponding to area upper the baseline $(0 \mathrm{mV})$. During the MVCs, EMG signals were quantified by using RMS, which was calculated over a 1-s period after the torque had reached a plateau. The EMG RMS was normalized to the $\mathrm{RMS}_{\mathrm{M}}$ by using the ratio RMS/RMS ${ }_{M}$, for both VL and VM muscles. A reduction in the EMG RMS without a reduction in $\mathrm{RMS}_{M}$ may be interpreted as a central activation failure.

Cycling exercise. All experiments were conducted on an electromagnetically braked cycle ergometer (Excalibur, Lode, Groningen, The Netherlands) where the seat and handlebars were fully adjustable both vertically and horizontally to replicate habitual positioning of subjects on their own bicycles. The ergometer was also equipped with racing pedals, with toe clips attached, which allowed subjects to wear cycling shoes. Pedaling rate was recorded instantaneously from the ergocycle by using a computer. The ergometer allowed subjects to keep power output constant independent of the pedal rate they naturally adopted. No feedback was given to the subjects concerning their self-selected cadence during the entire experiment. Inside air temperatures ranged from 21 to $23^{\circ} \mathrm{C}$. Two fans were placed in front of the ergocycle to reduce sweating during cycling. During the 5-h cycling exercise, subjects ingested a $6 \mathrm{~g} / 100 \mathrm{ml}$ glucose polymer solution at a rate of $1,000 \mathrm{ml} / \mathrm{h}$. This drinking regimen was intended to prevent hypoglycemia and dehydration.

\section{Protocol and Experimental Procedures}

Preliminary session. During an initial session that took place at least 5 days before the experiment, each of the nine subjects performed a continuous, incremental cycling test to determine their MAP. Briefly, the test began with a warm-up at $150 \mathrm{~W}$ for $5 \mathrm{~min}$, after which the power output was increased by increments of $25 \mathrm{~W}$ every 2 min until volitional exhaustion. MAP output was the highest power completed for $2 \min (381 \pm 37 \mathrm{~W})$. Thirty minutes after the incremental test, subjects familiarized themselves with the Biodex isokinetic measurement apparatus and the transcutaneous stimulation for strength testing. 
Rectangular protocol. The experiment consisted of sustaining $55 \%$ of MAP for 300 min (excluding the 10-min warm-up period) at a free pedaling rate. Data from previous studies $(18,26)$ suggest that $55 \%$ of MAP would be close to the maximal intensity that the subjects could maintain for 300 min. The power output was constant during the 5-h cycling exercise. Each hour, the subjects stopped the cycling exercise to perform the neuromuscular tests. This neuromuscular testing session lasted on average $<10 \mathrm{~min}$.

Neuromuscular performances. A standardized warm-up was carried out by each subject before the session. It consisted of 10 min of cycling at $33 \%$ of MAP, followed by four submaximal concentric knee extensions and two submaximal 3 -s isometric knee extensions. Subsequent to this warm-up protocol, the femoral nerve of the right leg was electrically stimulated at rest to obtain the M-wave and the twitch contractile properties. Three stimuli each separated by $5 \mathrm{~s}$ were given first. Subjects were then asked to perform two to three MVCs (4-5 s), and a single twitch was superimposed to the isometric plateau. Verbal encouragement was given to the subjects during all maximal contractions. A rest period of $40 \mathrm{~s}$ was permitted between each MVC to minimize the duration of the neuromuscular testing session each hour. A strict timing was respected to secure constant conditions among repeated measurements. The same experimental procedure (lasting $\sim 10 \mathrm{~min}$ ) was carried out before and after 60 $\min (\mathrm{H} 1), 120 \mathrm{~min}(\mathrm{H} 2), 180 \mathrm{~min}$ (H3), $240 \mathrm{~min}$ (H4) and 300 min (H5) of cycling and after $30 \mathrm{~min}$ of recovery.

Subjects had to indicate the perceived exertion value according to the Borg's scale of 6-20 (5) at the end of each hour. The rate of perceived exertion at the end of the exercise was $19 \pm 1$, indicating that the subjects had reached a high level of subjective fatigue.

\section{Statistical Analysis}

All data are expressed as means $\pm \mathrm{SD}$ (table) or $\pm \mathrm{SE}$ (figures). The data recorded during the exercise and recovery were statistically tested using a one-factor (time) ANOVA with repeated measures. When significant main effects were found, the Tukey test was used for post hoc analysis. Significance was accepted when $P<0.05$. The statistical analyses were undertaken by using Statistica software for Windows (StatSoft, version 5.1, Statistica, Tulsa, OK).

\section{RESULTS}

\section{Muscular Torque and Activation}

Figure $1 A$ shows that MVC torque was significantly $(P<0.01)$ reduced by the exercise. Compared with "before exercise" values, the mean MVC torque decreased by $9 \%$ after the first hour (H1) and remained at this level for the next $2 \mathrm{~h}(-8 \%$ at $\mathrm{H} 2,-10 \%$ at $\mathrm{H} 3)$. A second fall was observed at the end of the fourth hour (H4) where MVC dropped by $16 \%$ compared with the before exercise value. At the end of the exercise (H5), the subjects lost on average $18 \%$ of MVC. Lower MVC values after 30 min of recovery compared with preexercise values $(-16 \%)$ demonstrated that subjects had not recovered at this time.

RMS/RMS ${ }_{M}$ for both VL and VM muscles are shown in Fig. $1, B$ and $C$, respectively. For the VL muscle, RMS/RMS ${ }_{M}$ was significantly $(P<0.05)$ reduced by the exercise: it decreased by $16 \%$ after the first hour and remained significantly lower during the next $4 \mathrm{~h}(\mathrm{H} 2$ :
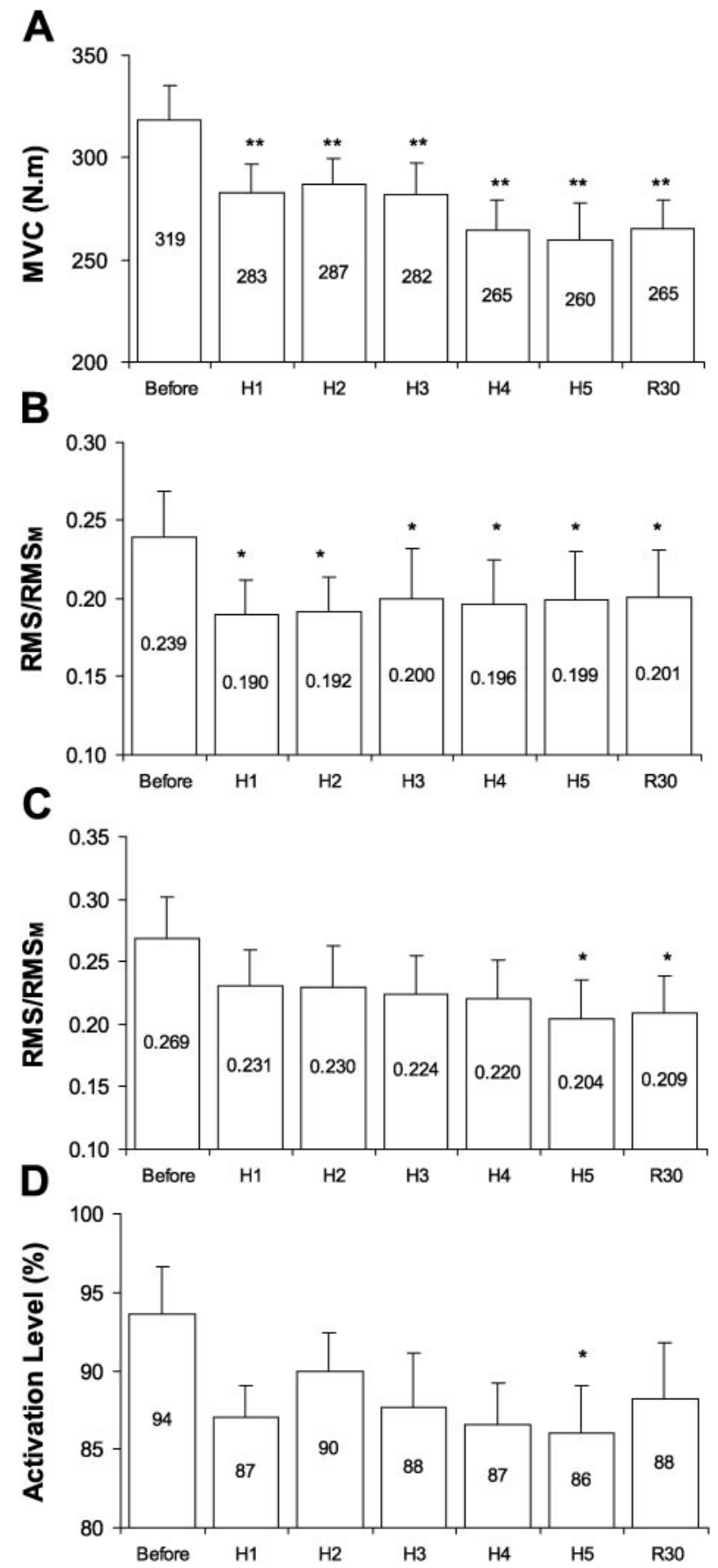

Fig. 1. Maximal voluntary contraction (MVC; $A$ ), electromyograph activity of vastus lateralis muscle $(B)$, electromyograph activity of vastus medialis muscle $(C)$, and activation level estimated by the superimposed twitch method $(D)$ before, during (H1: 60th min, H2: 120th min, H3: 180th min, H4: 240th min), immediately after (H5: 300th $\mathrm{min}$ ), and $30 \mathrm{~min}$ after (R30) the 5 -h cycling exercise. Values are means \pm SE. RMS, root mean square; $\mathrm{RMS}_{\mathrm{M}}$, RMS of M wave. Statistically significant compared with before exercise values: $* P<$ $0.05 ; * * P<0.01$

$-13 \%$, H3: $-12 \%, \mathrm{H} 4:-14 \%, \mathrm{H} 5:-14 \%)$, and after 30 min of recovery $(-12 \%)$. For the VM muscle, RMS/ $\mathrm{RMS}_{\mathrm{M}}$ decreased by $9 \%$ after the first hour and remained lower during the next $3 \mathrm{~h}$ (H2: $-9 \%, \mathrm{H} 3:-11 \%$, $\mathrm{H} 4:-12 \%)$. Nevertheless, the reduction of $\mathrm{RMS} / \mathrm{RMS}_{\mathrm{M}}$ 


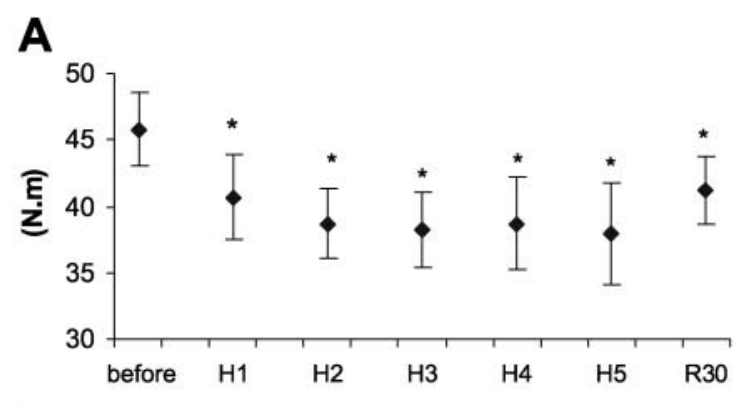

B

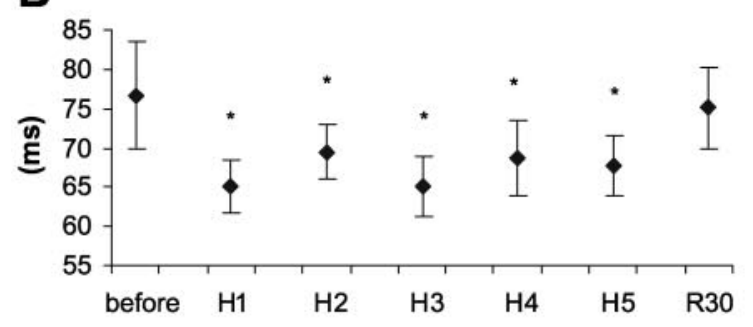

C

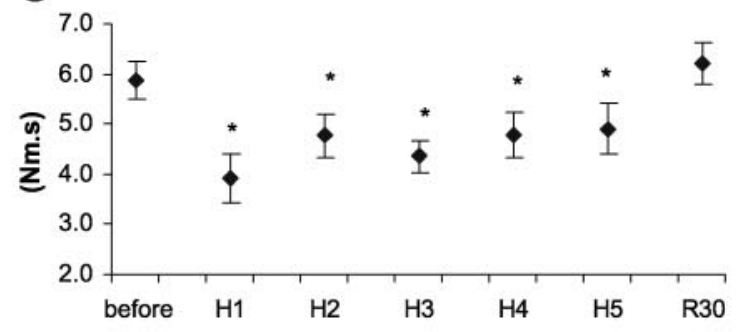

D

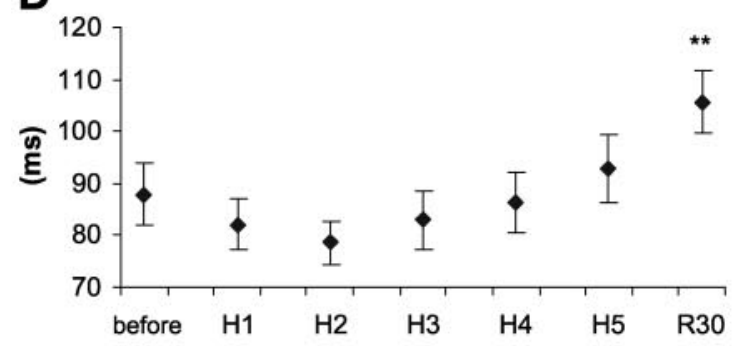

E

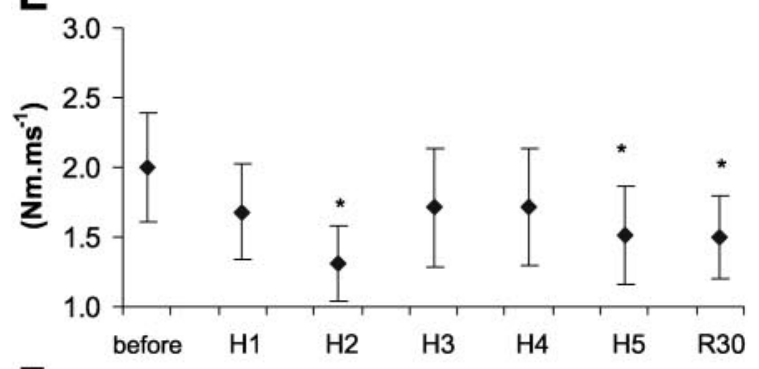

$\mathbf{F}$

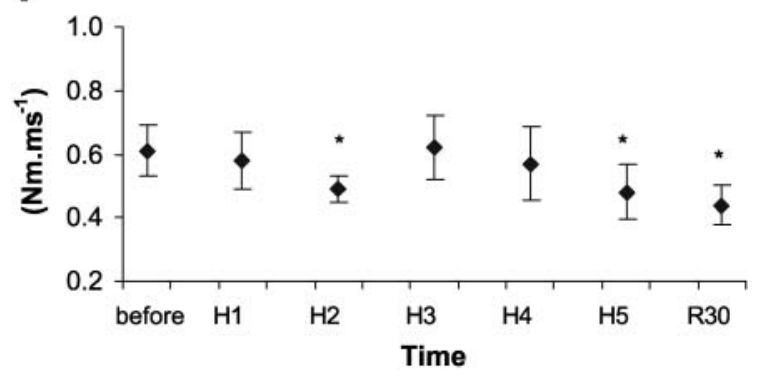

was only significant $(P<0.05)$ at the end of the exercise (H5) when it dropped down to $20 \%$ compared with the before exercise value. RMS/RMS ${ }_{M}$ for the VM muscle was still $18 \%$ lower $(P<0.05)$ after $30 \mathrm{~min}$ of recovery.

The central activation level estimated by the twitch interpolation method tended to fall by the first hour of exercise and remained lower for the rest of the cycling exercise and after $30 \mathrm{~min}$ of recovery (Fig. $1 D$ ). Nevertheless, because of the great intersubject variability, the reduction of the activation level was only significant $(P<0.05)$ at the end of exercise (H5).

\section{Twitch Contractile Properties and $M$ wave}

Changes in contractile parameters of the muscular twitch are shown in Fig. 2. $\mathrm{P}_{\mathrm{t}}$ decreased significantly $(P<0.05)$ during the exercise by $16 \pm 20 \%(45.8 \pm 8.2$ to $37.9 \pm 11.5 \mathrm{~N} \cdot \mathrm{m})$ and remained significantly lower $(P<0.05)$ after $30 \mathrm{~min}$ of recovery compared with the $\mathrm{P}_{\mathrm{t}}$ values before exercise. During the exercise, contraction time and total area of mechanical response also decreased significantly $(P<0.05)$, by $9 \pm 11$ and $16 \pm$ $19 \%$, respectively, but returned to values close to pre exercise after $30 \mathrm{~min}$ of recovery. Surprisingly, the half-relaxation time did not change during the exercise but showed a significant $(P<0.01)$ increase after 30 min of recovery when compared with before exercise values. Maximal rate of twitch torque development and relaxation tended to decrease but the difference compared with before-exercise values were only significant $(P<0.05)$ after 2 and $5 \mathrm{~h}$ of cycling and after $30 \mathrm{~min}$ of recovery.

The characteristics of $\mathrm{M}$ wave were minimally altered during the exercise (Table 1). The M-wave duration of VM muscle increased slightly during the exercise, but the increase was only significant $(P<0.05)$ after $4 \mathrm{~h}$ of exercise and remained high after $30 \mathrm{~min}$ of recovery. No significant increase was found for VL muscle except after 30 min of recovery $(P<0.05)$. The M-wave amplitude was not significantly altered by the exercise in either the VL or VM muscle. A significant decrease $(P<0.05)$ of the $\mathrm{RMS}_{\mathrm{M}}$ was found in VL muscle after $4 \mathrm{~h}$ and at the end of exercise. No significant changes in $\mathrm{RMS}_{\mathrm{M}}$ were found for the VM muscle.

\section{DISCUSSION}

The purpose of the present study was to examine the time course of changes in the neuromuscular (neural and contractile) properties of the quadriceps muscle during a 5-h cycling exercise performed at 55\% of MAP. Changes in peripheral mechanisms such as reduction in $\mathrm{P}_{\mathrm{t}}$, contraction time, and total twitch area were

Fig. 2. Twitch contractile properties of the quadriceps muscle before, during (H1, H2, H3, H4), immediately after (H5), and 30 min after (R30) the 5-h cycling exercise. A: maximal twitch torque. $B$ : contraction time. $C$ : total area of mechanical response. $D$ : half-relaxation time. $E$ : maximal rate of twitch torque development. $F$ : maximal rate of twitch torque relaxation. Values are means \pm SE. Significant differences compared with before-exercise values: ${ }^{*} P<0.05$; $* * P<$ 0.01 . 
Table 1. M-wave characteristics before, during (H1, H2, H3, H4), immediately after (H5), and 30 min after (R30) the 5-h cycling exercise for both vastus medialis and vastus lateralis muscles

\begin{tabular}{|c|c|c|c|c|c|c|}
\hline & \multicolumn{3}{|c|}{ Vastus Lateralis } & \multicolumn{3}{|c|}{ Vastus Medialis } \\
\hline Before & $9.01 \pm 1.57$ & $8.88 \pm 1.12$ & $2.77 \pm 0.43$ & $8.04 \pm 1.57$ & $8.24 \pm 2.73$ & $2.56 \pm 0.80$ \\
\hline $\mathrm{H} 2$ & $8.67 \pm 1.16$ & $9.29 \pm 1.44$ & $2.86 \pm 0.47$ & $8.98 \pm 2.59$ & $8.66 \pm 3.39$ & $2.78 \pm 1.15$ \\
\hline H3 & $9.34 \pm 1.24$ & $8.55 \pm 1.71$ & $2.72 \pm 0.64$ & $9.23 \pm 2.31$ & $8.64 \pm 3.82$ & $2.83 \pm 1.36$ \\
\hline $\mathrm{H} 4$ & $9.14 \pm 1.17$ & $8.22 \pm 2.04$ & $2.58 \pm 0.66^{*}$ & $9.45 \pm 2.11^{*}$ & $8.49 \pm 3.00$ & $2.62 \pm 0.95$ \\
\hline H5 & $9.58 \pm 1.78$ & $8.19 \pm 1.94$ & $2.55 \pm 0.57^{*}$ & $9.58 \pm 2.15^{*}$ & $7.95 \pm 2.85$ & $2.53 \pm 0.88$ \\
\hline
\end{tabular}

Values are means $\pm \mathrm{SD} . D$, duration; $A$, amplitude; $\mathrm{RMS}_{\mathrm{M}}$, root mean square of the M wave; H1, 60th min; H2, 120th min; H3, 180th min; H4, 240th min; H5, 300th min; R30, 30 min of recovery. * Significant differences compared with before-exercise values, $P<0.05$.

observed from the first hour of exercise. In contrast, M-wave properties representing muscle excitability were altered exclusively in the last part of the exercise. Whereas VL muscle EMG activity was significantly reduced from the first hour, EMG activity from VM muscle and central activation estimated by the twitch interpolation method were significantly lower at the end of the exercise compared with before.

Changes in muscle excitability during the 5 -h cycling exercise were assessed by examining of the characteristics of the $\mathrm{M}$ wave. In our experiment, the only significant changes in $\mathrm{M}$-wave characteristics were found during the last part of the cycling exercise. Indeed, after $4 \mathrm{~h}$ of exercise, $\mathrm{RMS}_{\mathrm{M}}$ of the VL muscle became significantly lower compared with preexercise. Also, M-wave duration of the VM muscle significantly increased, which may represent a decrease in conduction velocity along the muscle fibers. Such phenomenon is frequently observed with fatigue and can also be estimated by spectral compression analysis of the EMG signal (20). In the present study, a longer M-wave duration was anticipated because fatigue is known to induce $\mathrm{K}^{+}$and ammonia accumulation (21), which alters fiber excitability. This impairment of neuromuscular propagation resulting from alteration in ionic process $\left(\mathrm{Na}^{+}-\mathrm{K}^{+}\right.$pump, $\mathrm{Na}^{+}-\mathrm{K}^{+}$gradients $)$still persisted 30 min after the exercise, suggesting that muscle excitability was still altered. An increase in VL and VM M-wave duration has already been found in a previous experiment performed in our laboratory after a 2 -h cycling exercise (18). In contrast, a high-intensity 30-min cycling exercise did not alter the M-wave characteristics (19). This discrepancy is frequently reported in the literature and may be due, at least in part, to differences in tasks performed to induce fatigue.

In contrast to the $\mathrm{M}$ wave, the contractile properties were altered after the first hour of the exercise, indicating that alteration of the excitation-contraction coupling process took place early in the exercise. Because twitch torque and contraction time both decreased after the first hour, maximal rate of twitch torque development was less subject to significant variations, except at the end of exercise. Significant reductions in $\mathrm{P}_{\mathrm{t}}$, contraction time, and total area of the mechanical response still persisted through the 5 -h exercise. At the end of the exercise, all the contractile parameters were significantly altered except the half-relaxation time. Impairment of excitation-contraction coupling has already been observed after shorter cycling exercises at a higher intensity $(18,19)$, but, in contrast, Millet et al. (24) have reported an increase in twitch mechanical response after running an ultramarathon $(\sim 8 \mathrm{~h})$. The precise mechanism for the changes in twitch properties is not known, and several processes might be impaired, including a reduced $\mathrm{Ca}^{2+}$ release from sarcoplasmic reticulum and reduced capacity of cross bridges to form strong bonds (11). These alterations in turn may be due to metabolic changes induced by exercise (accumulation of $\mathrm{H}^{+}$and inorganic phosphate) $(10,22)$. The relative preservation of the half-relaxation time has already been reported by Lepers et al. (18) after a 2-h cycling exercise and by Booth et al. (4) after an exhausting cycling exercise lasting $\sim 1.5 \mathrm{~h}$. However, the half-relaxation time measured during recovery significantly increased. This may be due to a delayed impairment of the processes that govern the half-relaxation time i.e., $\mathrm{Ca}^{2+}$ movements.

The impairment of contractile properties of the quadriceps muscle could partially explain the decline of the maximal voluntary force observed throughout the 5 -h cycling exercise. Maximal voluntary torque at the knee joint progressively decreased during the exercise while the subjects were still able to maintain the desired power output on the ergocycle. Compared with preexercise values, the maximal isometric torque was significantly reduced by $18 \%$ at the end of exercise. The decreased force-generating capacity of the quadriceps muscle observed after cycling in the present study is in a similar range observed in previous studies after shorter and higher intensity cycling exercise $(2,17,18)$. Our laboratory previously found a reduction of $13 \%$ in MVC after either a 2 -h cycling exercise at $65 \%$ of MAP (18) or a 30-min cycling exercise at $80 \%$ of MAP (19). However, greater force reductions $(\sim 30 \%)$ were observed after cycling exercise lasting $<2 \mathrm{~h}$ but performed until exhaustion $(4,25)$.

In the present study, the first method adopted to investigate potential impairment in central drive was the ratio between the RMS values obtained during MVC and the RMS of the respective $\mathrm{M}$ wave (i.e., RMS/RMS ${ }_{M}$ ). For the VL muscle, the RMS/RMS ${ }_{M}$ ratio decreased significantly after the first hour of exercise, 
whereas the reduction for the VM muscle was only significant at the end of exercise. This reduction of muscle activation, responsible for the decrease in force production associated to muscle fatigue, has already been observed by several authors after prolonged cycling exercise $(2,18,19)$. St Clair Gibson et al. (26) recently found a progressive decrease in EMG activity of the rectus femoris muscle associated with a decrease in power output during repeated high-intensity exercise bouts performed during a $100-\mathrm{km}$ cycling time trials. These data support the reduction in muscle activation during the exercise observed in the present study. The faster decline of muscular activity during MVC for the VL muscle compared with the VM muscle is difficult to interpret. In contrast, our laboratory previously reported a significant RMS reduction for the VM muscle but not for the VL during MVC performed after a 2-h cycling exercise at $65 \%$ of MAP (18). It has been found that fatigue during isometric contractions may differ between monoarticular vs. biarticular agonist muscles (12). Our data suggest that a submaximal dynamic fatiguing exercise may differently affect muscle activation of monoarticular agonist muscles. Further studies examining muscular activity of synergist muscles during long-duration cyclic activity such as cycling are needed to better understand the changes in recruitment activity observed with fatigue.

The method of superimposed stimulation during MVC used here had been successfully included as part of research investigating the level of voluntary muscle activation of the quadriceps muscle $(2,19)$. From Fig. $1 D$, it follows that the subjects significantly decreased their activation level only at the end of the exercise. It is nevertheless interesting to note that decreases were observed after the first hour, but because of great interindividual variability, these values did not reach significance. A reduction in activation level estimated by the superimposed technique suggested that a reduced number of motor units was voluntarily recruited by the subjects at the end of the exercise. Nevertheless, the second mechanism for the reduction in central drive (i.e., decrease in motoneuron firing rates) cannot be ascertained by using the present methodology (23).

In this experiment, the two methods used for estimating central activation both attest that a central component of muscle fatigue contributes substantially to the reduction of maximal voluntary force, especially at the end of the prolonged cycling exercise. The reduced neural drive the motoneurons may be due to spinal and supraspinal mechanisms (for review, see Ref. 14). Fatigue can occur at levels upstream of corticospinal neurons, presumably due to neurotransmitter depletion, which could result in an impaired efficiency in generating the central command. Indeed, during prolonged exercise, the increase of lipolysis due to glycogen depletion is known to changes the plasma free tryptophan concentration and thus the brain free tryptophan which is a precursor of serotonin (9). Increase brain serotonin has been suspected to lead central fatigue by reducing the corticospinal impulses reaching the motoneurons and thus the central activation level (8). Central fatigue could also occur at the spinal level from peripheral reflex inhibition of the $\alpha$-motoneuron pool (17) and/or disfacilitation of the $\alpha$-motoneurons by muscle spindle afferents (3). Moreover, peripheral inhibitory input is thought to be conveyed from receptors sensitive to metabolic changes in exercising muscles via group III and IV afferents (16). Therefore, alterations of neuromuscular activity during such a prolonged exercise could be due to commands generated in the higher cortical structures and/or in response to afferent input from metabolic changes in the muscles.

In conclusion, this study examined the changes in neuromuscular properties of the quadriceps muscle occurring during a long-duration cycling exercise. The findings demonstrated that a 5 -h cycling exercise performed at $55 \%$ of the MAP progressively reduced the maximal voluntary force-generating capability in the quadriceps muscle. The time course was such that the contractile properties were significantly altered after the first hour, whereas excitability and central drive were more impaired toward the latter stages.

The authors thank Dr. Sandra Hunter for helpful suggestions concerning this manuscript.

\section{REFERENCES}

1. Bangsbo J, Graham TE, Kiens B, and Saltin B. Elevated muscle glycogen and anaerobic energy production during exhaustive exercise in man. J Physiol (Lond) 451: 205-227, 1992.

2. Bentley DJ, Smith PA, Davie AJ, and Zhou S. Muscle activation of the knee extensors following high intensity endurance exercise in cyclists. Eur J Appl Physiol 81: 297-302, 2000.

3. Bongiovanni LG and Hagbarth KE. Tonic vibration reflexes elicited during fatigue from maximal voluntary contractions in man. J Physiol (Lond) 423: 1-14, 1990.

4. Booth J, McKenna MJ, Ruell PA, Gwinn TH, Davis GM, Thompson MW, Harmer AR, Hunter SK, and Sutton JR. Impaired calcium pump function does not slow relaxation in human skeletal muscle after prolonged exercise. J Appl Physiol 83: 511-521, 1997.

5. Borg GAV. Perceived exertion as an indicator of somatic stress. Scand J Rehabil Med 2: 92-98, 1970.

6. Coyle EF, Coggan AR, Hemmert MK, and Ivy JL. Muscle glycogen utilization during prolonged strenuous exercise when fed carbohydrate. J Appl Physiol 61: 165-172, 1986.

7. Davies CTM and Thompson MW. Physiological responses to prolonged exercise in ultramarathon athletes. J Appl Physiol 61: 611-617, 1986.

8. Davis JM and Bailey SP. Possible mechanisms of central nervous system fatigue during exercise. Med Sci Sports Exerc 29: 45-57, 1997.

9. Davis JM, Bailey SP, Woods JA, Galiano FJ, Hamilton MT, and Bartoli WP. Effects of carbohydrate feedings on plasma free tryptophan and branched-chain amino acids during prolonged cycling. Eur J Appl Physiol 64: 513-519, 1992.

10. Donaldson SK, Hermansen L, and Bolles L. Differential, direct effects of $\mathrm{H}^{+}$on $\mathrm{Ca}^{2+}$-activated force of skinned fibers from soleus, cardiac and adductor magnus muscles of rabbits. Pflügers Arch 376: 55-65, 1978.

11. Duchateau J and Hainault K. Electrical and mechanical failures during sustained and intermittent contractions in humans. J Appl Physiol 58: 942-947, 1985.

12. Ebenbichler GR, Kollmitzer J, Glockler L, Bochdansky T, Kopf A, and Fialka V. The role of the biarticular agonist and cocontracting anatagonist pair in isometric muscle fatigue. Muscle Nerve 21: 1706-1713, 1998.

13. Fitts RH. Cellular mechanisms of muscle fatigue. Physiol Rev 74: 49-94, 1994. 
14. Gandevia SC. Spinal and supraspinal factors in human muscle fatigue. Physiol Rev 81: 7525-7589, 2001.

15. Gandevia SC, Herbert RD, and Leeper JB. Voluntary activation of human elbow flexor muscles during maximal concentric contractions. J Physiol (Lond) 512: 595-602, 1998.

16. Garland SJ and Kauffman MP. Role of muscle afferents in the inhibition of motoneurons during fatigue. In: Fatigue, edited by Gandevia SC, Allen GM, and McKenzie KK. New York: Plenum, 1995, p. 271-278.

17. Garland SJ, and McComas. Reflex inhibition of human soleus muscle during fatigue. J Physiol (Lond) 429: 17-27, 1990.

18. Lepers R, Hausswirth C, Maffiuletti NA, Brisswalter J, and Van Hoecke J. Evidence of neuromuscular fatigue after prolonged cycling exercise. Med Sci Sports Exerc 32: 1880-1886, 2000.

19. Lepers R, Millet G, and Maffiuletti NA. Effect of cycling cadence on contractile and neural properties of knee extensors. Med Sci Sports Exerc 33: 1882-1888, 2001.

20. Lowery MM, Vaughan CL, Nolan PJ, and O'Malley MJ. Spectral compression of the electromyographic signal due to decreasing muscle fiber conduction velocity. IEEE Trans Rehabil Eng 8: 353-361, 2000.

21. MacLaren DP, Gibson H, Parry-Billings M, and Edwards RHT. A review of metabolic and physiological factors in fatigue.
In: Exercise and Sports Sciences Reviews, edited by Pandolf KB. Baltimore, MD: Williams \& Wilkins, 1989, vol. 17, p. 29-66.

22. Metzger JM and Moss RL. pH modulation of the kinetics of a $\mathrm{Ca}^{2+}$ sensitive cross-bridge state transition in mammalian single skeletal muscle fibers. J Physiol (Lond) 428: 751-764, 1990.

23. Miller M, Downham D, and Lexall J. Superimposed single twitch and pulse train stimulation: a quantitative assessment during submaximal isometric knee extension in young, healthy men. Muscle Nerve 22: 1038-1046, 1999.

24. Millet GY, Lepers R, Maffiuletti NA, Babault N, Martin V, and Lattier G. Alterations of neuromuscular function after an ultramarathon. J Appl Physiol 92: 486-492, 2002.

25. Sahlin $\mathbf{K}$ and Seger JY. Effects of prolonged exercise on the contractile properties of human quadriceps muscle. Eur J Appl Physiol 71: 180-186, 1995.

26. St Clair Gibson A, Schabort EJ, and Noakes TD. Reduced neuromuscular activity and force generation during prolonged cycling. Am J Physiol Regulatory Integrative Comp Physiol 281: R187-R196, 2001.

27. Vercruyssen F, Hausswirth C, Smith D, and Brisswalter J. Effect of exercise duration on optimal pedaling rate choice in triathletes. Can J Appl Physiol 26: 44-54, 2001.

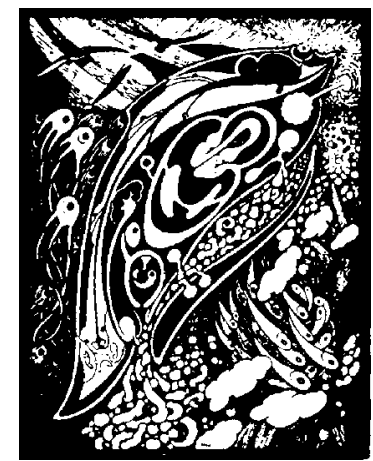

